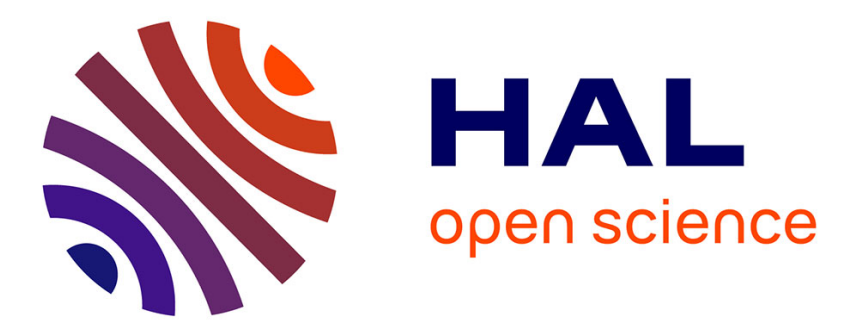

\title{
Rencontre avec une mère précarisée et ses " bébés précaires "
}

Rebecca Faura-Loussert, Marion Feldman

\section{To cite this version:}

Rebecca Faura-Loussert, Marion Feldman. Rencontre avec une mère précarisée et ses " bébés précaires ". La psychiatrie de l'enfant, 2019, 62 (2), pp.257-271. 10.3917/psye.622.0257 . hal-03131625

\section{HAL Id: hal-03131625 \\ https://hal.parisnanterre.fr/hal-03131625}

Submitted on 4 Feb 2021

HAL is a multi-disciplinary open access archive for the deposit and dissemination of scientific research documents, whether they are published or not. The documents may come from teaching and research institutions in France or abroad, or from public or private research centers.
L'archive ouverte pluridisciplinaire HAL, est destinée au dépôt et à la diffusion de documents scientifiques de niveau recherche, publiés ou non, émanant des établissements d'enseignement et de recherche français ou étrangers, des laboratoires publics ou privés. 


\title{
RENCONTRE AVEC UNE MĖRE PRÉCARISÉE ET SES «BÉBÉS PRÉCAIRES "
}

\author{
REBECCA FAURA-LOUSSERT ${ }^{1}$, MARION FELDMAN ${ }^{2}$
}

\begin{abstract}
RENCONTRE AVEC UNE MĖRE PRÉCARISÉE ET SES «BÉBÉS PRÉCAIRES 》
Cet article interroge l'influence d'un contexte social spécifique et ses conséquences psychiques chez les parents, sur le déploiement des relations précoces mère-enfants, sur la construction psychique individuelle des enfants en question, et plus largement sur la construction familiale. S'inscrivant dans une approche compréhensive, aucune hypothèse n'a été pré-établie, le but étant, au contraire, de faire émerger des hypothèses de fonctionnement à l'issue de l'analyse des données cliniques. Les données en question ayant été recueillies par une méthode d'observation participante au sein de l'hôpital de jour mère-enfant où sont pris en charge Mme B et son fils Ezra.
\end{abstract}

Mots-clés : Précarité sociale, relations précoces mère-enfant.

\section{A MEETING WITH A SOCIALLY VULNERABLE MOTHER AND HER "VULNERABLE BABIES"}

This article examines the influence of a specific social context and its psychological consequences on parents, on the development of early mother-child relationships, on the individual psychological construction of the children in question, and on family construction more broadly. We adopt a comprehensive approach with no pre-established hypothesis, our aim being for operating assumptions to emerge after analysis of the data. The data in question were collected using a participatory observation method in day hospital where Mrs. B and her son, Ezra, were treated.

Keywords: Social vulnerability, early mother-child relationships.

\section{LE CONTEXTE}

En l'espace de quelques années, la figure de la pauvreté a considérablement changé. L'homme seul, dormant sur les strapontins du métro et mendiant quelques pièces aux voyageurs généreux, n'en est qu'une des facettes. La plupart du temps invisibles dans l'espace social, plus nombreuses sont aujourd'hui les familles, ou les femmes seules avec des enfants mineurs (Guyavarch et Garcin, 2014). Tant et si bien qu'un pôle "Familles " a été mis en place au sein du Samu Social de Paris dès 2001 afin de répondre au mieux à la demande croissante d'hébergement de ces dernières. En effet, alors qu'en 1999 un peu moins de $15 \%$ de l'ensemble des nuits d'hébergements assumées

1. Psychologue-clinicienne, doctorante au laboratoire Clinique, psychanalyse, développement ClipsyD EA4430 (A2P), Université Paris Nanterre.

2. Professeure de psychopathologie psychanalytique - Université Paris Nanterre. Chercheure au laboratoire Clinique, psychanalyse, développement ClipsyD EA4430 (A2P). Psychologue-clinicienne. 
par le 115 de Paris concernait des familles, en 2012 le score atteint non moins de $83 \%$. Il aurait pu augmenter encore si des restrictions budgétaires datant de 2011 n'avaient pas obligé les agents à refuser certaines demandes. Le Samu Social considère, par ailleurs, qu'un seuil a été franchi en 2010 . Il a estimé à 10280 les familles sans logement en Ile-de-France, soit 35000 personnes dont 17660 enfants de moins de 13 ans (Cabut et Santi, 2017). Pour autant, il semblerait que "la lutte contre la précarité et ses effets sur la santé [pourtant démontrés de plus en plus finement par les neurosciences entre autres] ne semble pas une priorité pour les politiques publiques" (Ibid, p. 12). Trop peu de budgets sont alloués actuellement aux institutions de santé publique pour les rendre capables d'accueillir dans des conditions plus favorables cette population de familles en grande précarité sociale. Pourtant, nous les voyons arriver de plus en plus nombreuses dans nos différentes structures d'accueil et de soin.

Dans ces conditions, la rencontre avec ces familles nous bouscule. Parfois même, elle nous sidère, car alors, elle nous met face à un Réel : celui de la pauvreté et du dénuement. Celui de la solitude, au milieu du surpeuplement des chambres minuscules et souvent miteuses qu'on leur alloue, pourtant fort heureusement. Un Réel par définition impensable, et révoltant pour les travailleurs humanistes que nous sommes, et qui nous confronte à l'impuissance la plus totale.

Cette impuissance, souvent d'origine contre-transférentielle, répond à celle du Sujet en situation de précarité : une impuissance absolue qui fait écho à sa dépendance tout aussi absolue à la société dans laquelle il vit et de laquelle il est, la plupart du temps, mis de côté. Le Sujet en situation de précarité n'a rien et est dans l'incapacité de réaliser les actions qui lui permettraient de répondre à ses besoins. Il attend donc de la société qu'elle le fasse, mais de la société, il ne vient rien. Il est condamné à attendre et subir, de manière passive. Il est littéralement passivé (Green, 1999).

Le mal-être des soignants et des intervenants sociaux constitue, selon Jean Furtos (2008a, 2008c), l'un des premiers marqueurs de ce qu'il appelle la souffrance psychique d'origine sociale. Selon lui, toujours, ce mal-être fait partie intégrante de la "clinique de la précarité ", et même plus : cela en est une condition!

\section{PRÉCARITÉ SOCIALE : ÉTAT DES LIEUX DE LA LITTÉRATURE}

Notons qu'il existe, étonnamment, encore peu de travaux sur la précarité sociale et ses conséquences, proportionnellement à l'étendue et la complexité de la problématique. Ceux qui existent s'organisent pour la plupart principalement autour des recherches de J. Furtos.

Selon lui, la clinique de la précarité est une clinique de l'exclusion : celle du monde des hommes. Avec l'idée qu'être exclu du tissu social, c'est comme perdre ce qui supporte la vie psychique en donnant à chacun un statut et un droit d'existence au sein d'une communauté humaine. D'une certaine manière, c'est d'une forme de mort dont il s'agit.

Mais la précarité, c'est aussi, et peut-être surtout, une exclusion de soi-même. J. Furtos parle de syndrome d'auto-exclusion. C'est-à-dire s'exclure soi-même 
pour ne pas souffrir d'être exclu. S'empêcher de vivre pour survivre. "Se couper de soi pour sauver soi ", écrit René Roussillon (2005a, p. 226), du côté de ce que Nathalie Zaltzman a nommé, par ailleurs, la pulsion anarchiste (1999). C'est probablement dans la même perspective que R. Roussillon (2008b) écrit ailleurs que "quand le piège suppose l'unité, suppose la continuité d'être, la solution peut se trouver dans le renoncement à cette unité " (p. 137). C'est aussi une façon de devenir actif dans le processus d'exclusion, de ne plus le subir passivement, et ainsi de ne pas le souffrir ni le penser.

On est là du côté des défenses dites paradoxales qui marquent la déconstruction du narcissisme (Furtos, 2008b) et constituent tant bien que mal le dernier rempart avant l'effondrement et le marasme. Un narcissisme qui entraine dans sa chute une triple perte de confiance (Furtos, 2008a) : en soi, en l'autre et en l'avenir. En soi, parce que le sujet perd toute "dignité d'exister" (p. 13). En l'autre, par qui il ne se sent plus reconnu dans son existence de Sujet. En l'avenir qui, de fait, devient inquiétant, entrainant une difficulté dans la possibilité même de désirer, de se projeter et de rêver. Par-là, c'est la capacité de transmission intergénérationnelle qui est mise à mal.

Cela marque la bascule d'une précarité constitutionnelle normale et structurante, à une précarité exacerbée (Furtos, 2008a). N'étant plus palliée par le socius, ni contenue par "le berceau culturel"-qui plus est lorsque la précarité sociale succède à une expérience migratoire (Moro, 1998 ; Rochette 2008) - la précarité constitutionnelle normale et structurante n'est plus à même de générer des liens de confiance et de reconnaissance. $\mathrm{Ni}$ avec les autres ni avec soi-même. Ce qui constitue une véritable disparition, remarquablement traduite dans la déshabitation de soi (Meryglod, 2008). Le corps même en vient à perdre ses fonctions de "socle" et de "noyau intime". Le sujet ne s'y sent plus engagé affectivement dans un lien d'appartenance (Ferrant, 2004, p. 152). Une attention particulière est donc à porter au symptôme incurique qui donne à voir la façon singulière de certains sujets de ne pas habiter leur corps. Sachant que les difficultés matérielles liées à la situation de précarité ne suffisent pas à expliquer cette symptomatologie (Meryglod, 2008).

Dans ce contexte, la rencontre clinique avec le Réel de la précarité sociale dans une structure de Seine-Saint-Denis a été très marquante et immédiatement colorée d'éprouvés contre-transférentiels forts. Travaillant alors auprès de dyades mère-bébé en difficulté, c'est autour de ce temps si particulier de la vie psychique que de nombreuses questions se sont cristallisées : comment prendre soin d'un être naturellement précaire, lorsque l'on est soi-même précaire ? Comment faire naître un être à sa psyché, lorsque l'on déserte la sienne ? Et ainsi, quelles sont les conséquences de la précarité sociale sur le déploiement des relations précoces entre une mère et son bébé ?

\section{LA NÉCESSITÉ D’ÉCRIRE ET DE CHERCHER : ITINÉRAIRE D’UNE PSYCHOLOGUE EN SEINE-SAINT-DENIS}

Face à ce Réel, écrire et chercher permettent de relancer la machine à penser en se constituant comme autant d'objets distanciateurs, afin de ne pas laisser 
ce sentiment d'impuissance à l'état d'obstacle, voire, au contraire, d'en faire un levier thérapeutique par une meilleure compréhension du fonctionnement des relations mère-enfant dans un contexte social précaire.

C'est cette démarche qui nous a animée tout au long d'une année riche d'enseignements dans une unité d'accueil et de soin mère-enfant. La vocation de cette structure est de prendre en charge les relations précoces mère-bébé, et ainsi prévenir l'apparition ou l'installation de troubles psychopathologiques chez l'enfant. Une année durant laquelle nous avons notamment rencontré la famille B.

\section{POINT DE MÉTHODOLOGIE}

Notons en préambule de l'illustration clinique que nous avons pris le parti de ne pas poser d'hypothèses au préalable, mais de "laisser parler " la clinique. Nous avons ainsi proposé une démarche exploratoire, inductive, inspirée de la méthodologie d'analyse de données dites par "théorisation ancrée " (Paillé, 1994). Il s'agit d'une méthodologie d'analyse qualitative qui part des données brutes de terrain (énoncés d'observation, entretiens cliniques, comptes-rendus de réunions etc.) et vise à les interpréter, les associer et les modéliser pour les constituer progressivement en une théorie. Elle s'effectue en plusieurs étapes : la codification d'abord qui consiste en la nomination, la définition, la thématisation du corpus à analyser, quasiment au mot à mot. Ensuite, la catégorisation de ces codes en concepts. Les catégories, consolidées et devenues à la fois résultats et outils d'analyse, sont ensuite mises en relation les unes avec les autres. La nature des liens est explicitée (lien de cause/conséquence, etc.). Cette étape est suivie par celle dite de l'intégration, qui vise à délimiter l'objet précis de la recherche. Ce dernier devra constituer le fil conducteur, l'élément de continuité de l'analyse et de la restitution de cette analyse. Il est toutefois évident que les thèmes qui se dégagent sont multiples, nécessitant qu'on les hiérarchise et les articule : certains deviennent principaux tandis que d'autres sont secondaires, subsidiaires, consécutifs à un autre, faisant progressivement apparaitre la construction pyramidale qui sera modélisée lors de l'étape suivante. La modélisation consiste alors à reconstituer, avec le plus de fidélité possible, l'organisation et la dynamique des relations structurelles et fonctionnelles caractérisant un phénomène ou un système (par exemple le fonctionnement du système familial en situation de précarité sociale). Enfin, la théorisation, ultime étape de la méthode, vise principalement à renforcer la théorie émergente et à affaiblir les explications divergentes (Paillé, 1994).

C'est une méthode qui a d'abord été utilisée en sociologie et en anthropologie. S'en inspirer s'avère également, à notre sens, d'une grande richesse pour aller au plus près du vécu subjectif de nos participants dans les recherches en psychologie. D'autant qu'elle permet, très naturellement, d'intégrer d'autres systèmes de références et d'interprétation que la seule psychologie ou psychanalyse en fonction des codes, catégories ou systèmes qui apparaissent et se répètent. Pour cela, elle s'adapte de manière remarquable à une approche transculturelle et à sa "méthode complémentariste" de laquelle nous nous réclamons (Devereux, 1967). Autrement dit, il s'agit de se référer de manière 
obligatoire, mais non simultanée, à différentes disciplines pour appréhender un même objet de recherche. Généralement l'anthropologie et la psychanalyse, mais l'histoire, la sociologie, la géo-politique et autres, ont également tout leur intérêt.

Les analyses de la présente recherche se sont inspirées des quatre premières étapes de la méthodologie par théorisation ancrée : la codification, la catégorisation, la mise en relation et l'intégration. Elles ont été réalisées d'abord de manière longitudinale, puis transversale à partir de cinq observations de la famille $\mathrm{B}$ qui correspondent à cinq moments différents de la prise en charge.

Elles font ainsi apparaitre trois niveaux de compréhension qui, là encore, correspondent à ceux de la "méthode complémentariste" (Devereux, 1967) : un niveau intrapsychique ; un niveau intersubjectif ; et enfin un niveau collectif. Ce dernier, apparaissant à l'issue de l'analyse transversale, permet d'opérer une mise en perspective du rapport du Sujet à la société dans laquelle il baigne, et ses conséquences sur les deux autres niveaux précédemment cités.

\section{LA FAMILLE B, ILLUSTRATION CLINIQUE}

Mme B et Ezra, son petit garçon alors âgé de 15 mois, ont été adressés à l'unité à la suite d'une information préoccupante émise par la PMI ${ }^{1}$. Cette dernière s'inquiétait du manque de stimulation d'Ezra et de ses frères, Carlo et Raphaël, que nous rencontrons ponctuellement à l'occasion des vacances scolaires. Pour autant, la CRIP $^{2}$ n'a pas donné suite, mettant en avant la grande précarité de la famille.

En effet, la famille B vit dans une situation de grande précarité sociale. Originaires d'Afrique subsaharienne, Mme B et son mari sont arrivés en France trois ans auparavant et ont très vite été déboutés du droit d'asile. L'impossibilité d'obtenir des papiers d'identité, entre autres, leur fait rencontrer de manière quotidienne des difficultés pour répondre à leurs besoins primaires que sont se loger, se vêtir et se nourrir.

\section{CINQ OBSERVATIONS : LES PRINCIPAUX THÈMES APPARUS AU TERME DE L'ANALYSE LONGITUDINALE}

\section{MME B, UNE PRÉSENCE ÉTRANGEMENT VIDE}

Dès la première observation, le thème de la disparition, à travers celui de l'absence, apparaît de manière récurrente. On en trouve des occurrences qui se déclinent sur différentes modalités, aussi bien chez Mme B que chez ses enfants, notamment chez Carlo, l'aîné alors âgé de 6 ans, et Raphaël, le cadet, âgé de 3 ans.

1. Protection maternelle et infantile.

2. Cellule de recueil des informations préoccupantes. 
En ce qui concerne Mme B, l'occurrence la plus marquante reste la suivante :

Alors que je ${ }^{1}$ suis assise par terre avec Ezra, aux pieds de Mme B, et que nous jouons avec une ardoise magique, je l'interpelle pour la faire participer. Je note alors que "Mme B ne réagit que peu, voire pas ». Ce que, contre-transférentiellement parlant, je vis de manière assez violente, car alors je ne trouve aucun écho, comme si elle n'était pas vraiment là et que, par ricochet, je n'existais pas vraiment là où elle se trouvait... Ainsi, elle est présente physiquement, mais comme absentée psychiquement.

Ce que nous décrivons comme " une présence/absence. Une présence étrangement vide. Comme un trou noir sans visage" peut rapidement évoquer la mère morte de Green (1983). Cette mère bien présente physiquement, pourvoyeuse de soins, mais absente psychiquement, c'est-à-dire rendue indisponible, de manière plus ou moins durable dans le temps, par des préoccupations (le plus souvent) de l'ordre de la dépression.

\section{DE LA PRÉSENCE ÉTRANGEMENT VIDE DE MME B À LA DISPARITION DE SES ENFANTS}

Cette modalité de présence de sa mère permet d'éclairer d'un sens tout à fait spécifique ce dont nous rend témoin Raphaël. Alors qu'il est installé pour le repas à une petite table, dos à $M m e$, il se retourne et dit "elle est là " (présence), avant de se tourner de nouveau face à son assiette en disant "elle est pas là " (absence). Certes, il peut s'agir là d'une tentative de symbolisation, par le "jeu ", de l'alternance entre présence et absence de la mère, comme une sorte de Fort-Da. Néanmoins la dimension contre-transférentielle, qui apparaît ici comme une boussole essentielle, suggère une autre orientation à l'interprétation.

En effet, j'écris que cela me "donnait l'impression que pour lui, à ce moment-là en tout cas, elle disparaissait [réellement] dès qu'il la perdait de vue."

Cette observation interroge ce que les psychologues du développement ont appelé "la permanence de l'objet " et qui est normalement acquise entre 18 et 24 mois (Raphaël a 3 ans), c'est-à-dire la possibilité de comprendre que l'autre existe toujours, même lorsqu'on le perd de vue. Ainsi son existence n'est pas simplement le fait de la perception que l'on a de lui. Ce n'est pas parce que nous ne voyons pas l'objet qu'il n'existe pas ou qu'il est absent. En des termes plus psychanalytiques, ce qui interroge ici, c'est ce que Raphaël a pu internaliser de sa mère. Une présence absente ? Un vide à l'endroit de la mère ? Il est question ainsi de sa capacité à garder, à l'intérieur de lui, une représentation vivante des objets qui perdure dans le temps. Une capacité, ou une incapacité peut-être, dont il nous donne une autre illustration, semble-t-il, le jour même lorsqu'en arrivant dans l'institution il fait le tour des soignants et nous demande l'un après l'autre qui nous sommes, quelle est notre identité ("comment tu t'appelles ?»). Lorsqu'une des psychologues revient après une courte absence, Raphaël lui demande de nouveau qui elle est. Comme si cela n'avait pas fait trace, et qu'ainsi aucun lien entre la femme qu'il avait rencon- 
trée en arrivant et celle qui venait de réapparaître ne pouvait être fait. Les objets semblent apparaitre et disparaitre sans qu'une continuité, une permanence puisse être manifestement établie, mettant en question la continuité interne même de Raphaël, et donc son sentiment d'existence. Raphaël se sent-il exister de manière continue ? À ce moment précis, se sent-il même exister tout simplement, ou agit-il en lui une forme de disparition, d'extinction ? C'est en tout cas une question que l'on peut légitimement se poser lorsque l'on relève les indices d'une adhésivité certaine, notamment à travers des comportements de collage "à la surface d'objets durs animés ou inanimés qui témoignent de l'utilisation défensive du processus d'identification adhésive " (Ciccone et Lhopital, 2001, p. 109). Deux thèmes apparaissent dans les données cliniques : celui de l'individuation, ainsi que celui de l'indifférenciation. Ce dernier fait particulièrement écho à la confusion entre l'objet et le moi qu'entraîne nécessairement l'identification adhésive qui ne souffre d'aucune solution de continuité entre l'un et l'autre (Ibid.).

Carlo, l'aîné, donne lui aussi à expérimenter des vécus d'absence/présence, et même de disparition. À plusieurs reprises, il "disparaît ", littéralement.

La première fois, alors que je me montre peu disponible pour lui, occupée à explorer un jouet musical avec Ezra, son petit frère. La seconde fois, à table, alors que j'accompagnais le repas de Raphaël, compliqué par un débordement d'excitation qu'il fallait contenir, aussi bien physiquement que psychiquement. J'écris ainsi que cela me donnait "l'impression de scènes desquelles il disparaissait faute d'attention ", faute d'un écho qu'il ne trouve pas chez moi, trop occupée ailleurs.

On peut interroger ici ce qui se répète, dans la dynamique transféro-contretransférentielle, du lien à une mère psychiquement indisponible chez laquelle on ne trouve que peu d'écho à sa propre existence, engendrant pour soi-même une certaine forme de disparition. On en revient ainsi à la question du sentiment continu d'existence, qui semble s'acter ici par une présence physique discontinue.

Où était Carlo, et que faisait-il dans ces moments-là ? Je ne saurais le dire, je n'en ai littéralement aucune représentation...

\section{«UN POUR TOUS, ET TOUS POUR UN" : HISTOIRE D’UNE DIFFICILE DIFFÉRENCIATION}

Les repas posent également d'autres questions. En effet, Mme B présente des conduites tout à fait singulières, et qui se répètent dans le temps : pour chaque cuillère que Mme B prépare pour Ezra, son benjamin, elle en prélève une petite partie.

«Dans tous les cas, écris-je encore, ça passe toujours d'abord par elle, par sa langue ». Pourrait-on percevoir cela comme pris dans une sorte de continuité des corps ?

Que cela nous expose-t-il d'une éventuelle difficulté de Mme B à voir ses enfants s'individuer ? Difficulté qui n'est pas forcément à mettre d'emblée du côté du pathologique. Toutefois, malgré le temps qui passe, ces conduites ne cèdent pas chez Mme B. Dans tous les cas, considérant à la suite d'Albert Ciccone (2012) que les contenus des observations ne sont pas de 
simples contenus en soi, mais des contenus toujours adressés au clinicien, il paraît juste ici d'en proposer tout de même une interprétation.

En l'occurrence, nous avons perçu cela comme une certaine forme d'indistinction des corps. Indistinction qui ferait que les limites entre ce qui appartient à l'un et ce qui appartient à l'autre, ce qui est adapté à l'un et ce qui est adapté à l'autre, seraient floues. Notons bien ici que les différentes interprétations ne s'excluent pas l'une l'autre, mais correspondent plutôt à différents niveaux de l'analyse renvoyant à des "strates" différentes du psychisme, tant individuel que dyadique ou familial. On voit bien ainsi comment s'articulent les différents espaces psychiques et comment les conséquences d'une situation donnée - sociale ici - peuvent se manifester en différents endroits, de différentes manières.

Cette orientation interprétative prend, quoi qu'il en soit, d'autant plus de puissance que la famille nous a donné d'autres indices allant dans le sens de l'indifférenciation.

Indifférenciation des espaces par exemple. Notamment lorsque Carlo construit une maison à l'aide de LEGO. Sa bâtisse est alors « faite de murs parallèles les uns aux autres, sans que rien ne soit fermé. Il les collait au maximum, laissant un espace minimal pour le passage des habitants. Les pièces n'étaient pas différenciées et s'interpénétraient les unes les autres, la salle de bain et les toilettes surtout, trônant en plein milieu ". Cette construction questionne par ailleurs la place de l'analité, et plus largement de la sphinctérisation, dans une fratrie dont les deux aînés ont présenté ou présentent encore des symptômes énurétiques.

Mais aussi, comme en miroir semble-t-il, indifférenciation des corps. En effet, «malgré l'espace [...] à leur disposition à l'unité, ils restent collés les uns aux autres devant le garage [entrer, sortir/ouvrir, fermer]. Ezra, le bras droit posé sur la jambe de Carlo, Raphaël à sa gauche».

Remarquons que dans la formulation même de la phrase ici, dont il faut avoir en tête toute la dimension subjective bien évidemment, il est déjà difficile de comprendre à qui appartient quel bras, quelle jambe, et qui se trouve où par rapport aux autres spatialement. C'est comme s'ils fonctionnaient comme un seul et même corps. D'ailleurs, on s'aperçoit que ce n'est qu'en associant ce que nous donne à voir chacun des enfants séparément que l'on arrive à se faire une représentation d'un fonctionnement qui pourrait appartenir à chacun d'eux en propre, comme le fonctionnement d'un seul et unique appareil psychique.

Là en tout cas, c'est d'un corps dont il semble s'agir. Un corps qui ne manquera pas, peu de temps après, suite à une augmentation massive et subite de l'excitation, de se démanteler, de s'éparpiller de part et d'autre de la pièce, chacun des enfants s'occupant dorénavant d'activités différentes les uns des autres.

\section{SYMPTÔMES ÉNURÉTIQUES ET DÉSORGANISATION TEMPORELLE}

Arrêtons-nous maintenant sur les symptômes énurétiques que nous évoquions plus tôt. Plus particulièrement, ceux de Raphaël.

$\mathrm{Au}$ cours des différentes observations, il est apparu que Raphaël pouvait s'uriner dessus, plusieurs fois successivement, dans des moments de jeu ou 
d'intense excitation. Ce qui interroge d'emblée, pour ce garçon, la possibilité dans ces moments de maintenir en soi le flux, de le contenir. Que cela nous apprend-t-il de sa capacité de contenance au niveau psychique ? Littéralement, ça déborde, ça le déborde. L'excitation aussi bien que l'urine, dans une correspondance corps-psyché qui voudrait, semble-t-il, que la quantité psychique trouve son écho dans la quantité corporelle. Ce qui ne peut être traité psychiquement est déchargé au niveau corporel, via la fonction sphinctérienne de la miction. Et plus précisément, le trouble de cette même fonction se manifestant par le symptôme énurétique.

Notons que le contrôle sphinctérien, qui fait défaut ici, ne relève pas uniquement d'un processus d'apprentissage physiologique. Il est foncièrement marqué par de nombreux processus psychiques qui, dans l'idéal, doivent permettre d'organiser les sensations provenant de la vessie et son état de tension (entre vide et pleine). Et ce, en fonction du déroulement du temps, pour ainsi anticiper la satisfaction du besoin (Schmit et Soulé, 2004) : "Cette activité de contrôle est donc liée à l'organisation temporelle $d u$ sujet ${ }^{1}$, et à sa perception de la durée qui est perturbée ou déniée chez l'énurétique»(p. 1752).

Effectivement, il est apparu au fil des observations que la temporalité dans la famille B est "organisée " de manière tout à fait particulière. Elle semble même plus désorganisée qu'organisée, à vrai dire. On le constate notamment dans la forme que prend la prise de notes de l'observatrice, marquée d'une certaine discontinuité. Surtout lorsqu'il s'agit de Raphaël, où il ne semble plus y avoir de véritable cohérence chronologique. La seule cohérence sur laquelle nous pouvons alors nous appuyer n'est pas chrono, mais associative. Elle attire ainsi notre attention sur une séquence autour d'un jouet musical frappé rythmiquement. Il s'agit, semblerait-il, d'une tentative de remettre du rythme, quelque chose qui vient égrener le temps de manière logique et continue, là où tout paraît désorganisé, illogique et discontinu. Remettre de l'ordre dans un monde vécu par la clinicienne comme en désordre. Cette observation peut être mise en lien avec ce que rapporte R. Roussillon (2005b) à propos des " toxicomanes de la musique ", qui s'imposent, par un biais extérieur, un rythme harmonieux. Ce dernier visant à contre-investir un vécu interne inverse de dysrythmie, et donc de chaos. "Il s'agit, ajoute-t-il, d'une forme particulière d'enveloppe ou de conteneur sonore utilisé comme système "pare-excitation" de prothèse" (p. 205). Or, en évoquant un "pare-excitation de prothèse ", c'est par contraste un pare-excitation qui fait défaut dont il est question. Nous y voilà donc.

\section{SUR LA PISTE DU PARE-EXCITATION PERDU}

Bien après Sigmund Freud qui l'avait déjà souligné, c'est D.W. Winnicott (1957) qui reviendra sur la fonction pare-excitante de l'objet externe, en l'occurrence la mère. À travers ce qu'il appelle l'object-presenting, cette dernière présente le monde à son enfant par petites doses. Des doses suffisamment petites et fractionnées pour que l'enfant puisse les traiter et que la découverte du monde se fasse de manière non traumatique. Or c'est bien cette notion de petites doses qui semble faire défaut à $\mathrm{Mme} B$, comme elle nous en rendra 
témoin notamment lors des moments de nourrissage, interrogeant sa propre capacité pare-excitante.

Lors de deux séquences différentes en effet, il apparait que les cuillères que prépare Mme B pour Ezra sont trop grosses ("énormes »), que le verre est trop plein ("rempli à ras bord 》). Autrement dit, les quantités sont inadaptées. Ce qui entraine d'ailleurs un contre-transfert fort, marqué par le malaise chez l’observatrice qui se dit "gênée pour Ezra pendant le repas ".

À cette difficulté de régulation chez la mère répond immédiatement la même fonction supportée par les soignants au sein du cadre du dispositif qui le permet (clinique $\mathrm{du}$ holding, faire-avec). Ainsi, nous régulons les quantités, les limitons en les fractionnant : de la vaisselle adaptée est fournie, le verre à bec est rempli "au quart ".

Le tout évoque une fonction pare-excitante, non pas tant psychique que littéralement à ras le corps, ne pouvant être assumée par Mme B à ce momentlà. Elle-même étant peut-être aux prises avec une excitation difficilement traitable. En effet, Mme B est dans une situation de précarité telle qu'il lui arrive d'avoir faim, de manquer de nourriture, là où l'Hôpital-de-Jour se fait le lieu d'une certaine opulence en comparaison de son dénuement. Alors que d'habitude il n'y a pas assez, soudain il y en a peut-être trop, ce qui peut rendre la balance difficile à faire.

Nous remarquons par ailleurs que la régulation des quantités de nourritures, leur fragmentation qui en fait des quantités adaptées à Ezra - autrement dit, traitables - ouvre sur la possibilité nouvelle d'un moment ludique. Un moment autour du besoin oral, durant lequel on ne différencie plus la satisfaction du besoin à proprement dit, du jeu; où s'arrête l'un et où commence l'autre. L'observatrice se montre d'ailleurs incapable de choisir entre les deux termes, comme s'il s'agissait de toute manière d'un entre-deux, d'une aire intermédiaire.

J'écris en effet, à propos du verre à bec remplit au quart : «Ezra s'en saisit et a bu/ joué avec".

Enfin, l'observation des moments de nourrissage fait également apparaître un jeu entre passivité et passivation, souvent pris dans le rapport à l'alimentation, et plus globalement à l'oralité. On voit notamment comment, à une certaine passivité chez Mme B, répond une passivation de Ezra ; passivation que l'on retrouve également dans les relations de soins qu'elle entretient avec Raphaël et Carlo à qui, par exemple, elle continue de brosser les dents. Carlo a pourtant 6 ans...

\section{ANALYSE TRANSVERSALE ET ÉLÉMENTS D’HYPOTHÈSE}

De l'analyse transversale, il ressortira différents axes de réflexion mettant en lien le rapport du sujet adulte à la société et ses conséquences d'un point de vue psychologique, avec les thèmes dévoilés par les analyses longitudinales. Nous en développerons trois. 
Nous nous apercevons qu'au défaut de la fonction pare-excitante de Mme B auprès de ses enfants, et notamment dans sa relation de nourrissage (Deronzier, 2015) avec Ezra, répond chez elle une difficulté à contrôler sa propre excitation. Une difficulté dont on peut supposer, à un autre niveau de l'analyse, qu'elle est en lien avec une fonction pare-excitante normalement assurée par une enveloppe psychique groupale, sociétale, culturelle, qui ferait là encore défaut. En effet, comme le rappelle René Kaës (2014), conceptualisée à partir des notions d'enveloppe psychique et de Moi-Peau d'Esther Bick et Didier Anzieu respectivement, la notion d'enveloppe psychique groupale est définie comme "produite par le groupe, nécessaire à son identité, capable de représenter les limites et les passages sélectifs entre le dedans et le dehors" (p. 50). Elle a pour fonction la maintenance psychique, la contenance psychique ainsi que celle de filtre et de pare-excitation. Or, comment est-il possible d'en bénéficier, lorsque l'on est exclu du dit groupe? Ou plus exactement lorsque l'on y est inclus, en tant qu'exclu - à exclure. Et qu'en ce sens, plus que du groupe social, c'est du groupe humain dont on est expulsé. Expulsion qui entraine, comme nous l'évoquions plus tôt, une triple perte de confiance. L'environnement devient alors menaçant et la rencontre avec lui, qui n'est plus médiatisée - fractionnée et limitée - par le socius, devient traumatique. Ce qui peut entraîner des états de confusion ou de perplexité chez le sujet et rendre ainsi, pour boucler la boucle, la fonction d'object presenting - par définition pare-excitante - de la mère en situation de précarité et d'exclusion, défaillante. En effet, comment peut-on présenter le monde de manière non traumatique, c'est-à-dire à petites doses, à son enfant, lorsque soi-même on ne cesse de le rencontrer de manière toujours plus traumatique?

\section{DU CORPS D'EXCEPTION DANS LA SOCIÉTÉ À L'INDIFFÉRENCIATION DES CORPS DANS LA FAMILLE}

Malika Mansouri et Hervé Bentata (2012) redéfinissent le corps d'exception comme cette façon que l'on a d'affilier à un corps unique et monolithique, "fabriqué " artificiellement par des mesures politiques et sociales exceptionnelles, un ensemble de personnes pourtant hétérogène. On abrase ainsi cette hétérogénéité qui signe la singularité de chacune des parties de ce tout douteux (Ibid). De cette façon, on inclut dans le corps social, en tant qu'il est exclu, ce corps exceptionnel.

Ce corps exceptionnel est un "groupe " artificiellement constitué et homogénéisé par ceux-là même qui excluent sur la base de ce qui leur apparait comme une altérité radicale. Ainsi, les individus composant ce "groupe " ne sont pas considérés pour eux-mêmes, avec leurs caractéristiques propres et individuelles. Ils sont plutôt comme les "fragments " d'un tout auquel ils ne peuvent s'identifier du fait d'un manque de communauté, si ce n'est celle de l'expérience de la précarité. Pour autant, il n'y a pas deux manières similaires d'être précarisé ; ce n'est pas une identité à part entière mais une condition sociale. L'amalgame est réducteur et enfermant. Il emprisonne dans une sorte de "magma indifférencié " ( $I d$, p. 118) dont les limites entre dedans et dehors ne peuvent 
être que floues et mouvantes, mettant à mal, par là même, toute possibilité d'exercer une fonction de filtre.

On assiste alors à une dissolution de la singularité de chacun, leur imposant une affiliation entre-soi complètement artificielle. Mansouri et Bentata (2012) parlent, de fait, d'une "indistinction massifiante " dont la violence tient à l'effacement progressif de la différence subjective. Or, il est intéressant de constater que cette indistinction propre au corps d'exception tend à se rejouer au sein de la famille $\mathrm{B}$, notamment dans les rapports qu'entretiennent entre eux les trois garçons. En effet, à travers l'indifférenciation des espaces d'abord, puis des corps ensuite, ils nous donnent l'impression de fonctionner comme un seul et unique organisme. Chacun y agit comme le fragment d'une totalité, d'une unité aliénante, dans laquelle, par définition, toutes les différences individuelles seraient abrasées. On ne distingue plus qui est l'un de l'autre, ni ce qui fait leur unicité.

Il y a même plus. Au-delà de ce que les enfants donnent à voir, c'est la forme même de l'analyse qui est imprégnée de cette abrasion des différences. En effet, il apparaît que les interprétations figurent une forme de continuité entre les trois enfants en ne prenant pas en compte les rapports différenciés qu'ils entretiennent avec Mme B, et que Mme B entretient avec eux. Tout est mis au même niveau d'analyse, comme si les relations de Mme B avec Ezra étaient identiques, symétriques, à celles qu'elle entretient avec Raphaël, ou avec Carlo. Ce qui n'est bien évidemment pas le cas. Et ce n'est qu'au prix d'un éclatement, d'une dispersion dans l'espace que les subjectivités peuvent de nouveau être perçues et pensées comme telles par l'observatrice. Ce dont il est question ici - et c'est important, nous semble-t-il, pour penser la clinique de la précarité - c'est du contre-transfert culturel. Autrement dit, la manière dont le thérapeute, l'observateur ou le chercheur, réagit intérieurement, à partir de ses propres représentations, préjugés et stéréotypes, à tout ce qui fait l'être culturel du patient, ou du sujet de la recherche (Moro, 2002). L'être culturel d'un "autre-étranger " perçu comme "différent ", du fait de son accent, de sa couleur de peau, de sa manière de se vêtir ou de faire, de son milieu socioéconomique, etc. Ces réactions sont obligatoires dans la mesure où tout thérapeute, chercheur ou observateur que nous sommes, nous sommes avant tout des êtres culturels. Nous sommes, de fait, baignés de représentations collectives culturellement codées, réinterprétées selon nos propres complexes, qui nous permettent de donner du sens à ce que l'on perçoit et ce que l'on vit. En ce sens, ces réactions sont également nécessaires (Rouchon et al., 2009). Ne pas les prendre en compte, et ne pas les analyser, ajoute ainsi au risque de passages à l'acte agressifs, affectifs ou racistes dans la prise en charge thérapeutique (Moro, 2002) ; et d'analyses qui concerneraient plus les représentations du chercheur que le vécu subjectif de ses participants. Dans le cadre de la recherche qui nous occupe, on se rend rapidement compte, en effet, de la manière dont la famille est perçue et dont les relations sont analysées avec une réelle imprégnation de représentations collectives internalisées et inconscientes de l'observatrice. Ce phénomène mis au jour et utilisé comme un outil supplémentaire d'interprétation, l'analyse n'en devient que plus riche. 


\section{D'UNE MÈRE PASSIVÉE À UNE MÈRE PASSIVANTE}

La relation de passivation qu'entretient Mme B avec ses enfants peut également être interrogée. En effet, nous avons constaté les difficultés qu'elle pouvait rencontrer à laisser faire ses enfants seuls pour eux-mêmes, notamment dans les soins primaires d'hygiène et de nourrissage. Ainsi, elle semble parfois les mettre dans une position où ils n'ont d'autre choix que d'être passifs. Elle fait pour eux, à leur place, les privant de la jouissance d'être actifs dans la satisfaction de leurs propres besoins. Ce qui peut s'envisager comme un renversement, en une position active, de la position de passivité qu'elle présente par ailleurs. Une position de passivité où il apparait que Mme B a besoin d'un tiers, institutionnel, qui agisse à sa place, qui fasse pour elle ; une position dont on est en droit d'interroger la nature. Serait-ce là le signe que Mme B a fini par s'identifier à une position de passivation dans laquelle elle se sent mise par la société ? En effet, du fait de sa situation sociale, mais aussi administrative - Mme B n'a pas de papiers - elle est nécessairement mise dans une situation d'impuissance quasiment absolue à réaliser les actions - et donc à être active qui lui permettraient de répondre à ses besoins et à ceux de sa famille. Elle est ainsi dans la position de dépendre des autres, d'une société qui pourtant l'exclut, pour obtenir ce dont elle a besoin. Littéralement, elle est dans l'impasse, elle ne peut rien faire d'autre qu'attendre que cela vienne d'un autre, représentant de la société, qu'il s'agisse de l'assistante sociale, ou des banques alimentaires. Elle ne peut rien faire sinon subir, ou bricoler, marchander mais au risque de tout perdre. Perdre ce qu'elle n'a pas. Ce qui fait écho à l'état de désaide, à cette sensation que pour survivre il faut tout attendre des autres, mais que des autres, justement, il ne vient rien. Le sujet est face à sa seule impuissance, sans recours, et ainsi dans une détresse certaine qui rappelle de manière on ne peut plus criante la situation traumatique dont elle est le prototype.

C'est à la suite de l'apparition de ces axes dans l'analyse transversale que nous avons émis l'hypothèse suivante : il semble que la relation qu'entretient Mme B avec la société et ses conséquences psychiques se reproduise, se répète, dans les rapports qu'elle instaure avec ses enfants. Les conséquences d'une telle modalité relationnelle mère-enfant font ricochet dans leur propre construction psychique individuelle, ainsi que dans la construction familiale.

Toutefois, il est clair que cette hypothèse peut être discutée, notamment en regard de nouveaux éléments cliniques de l'histoire de Mme B qui apparaissent dans une deuxième série d'observations. Des éléments qui semblent ne pouvoir poindre seulement parce que, entendu et accueilli, il y a quelque chose de l'iciet-maintenant de la précarité qui peut enfin céder. Filtrent alors des fragments d'une histoire passée qui va au-delà, et qui vient donner un sens nouveau (sans pour autant exclure l'autre, bien au contraire). C'est d'ailleurs là que l'on commence à voir apparaître quelque chose du processus thérapeutique.

\section{CONCLUSION : UNE RELATION MÈRE-SOCIÉTÉ À SOIGNER}

L'analyse du processus thérapeutique nous donne à voir et à vivre une progressive réhumanisation, une renarcissisation de Mme B. Les symptômes incuriques cèdent peu à peu, ou du moins se transforment. Cela passe notamment 
par le regard. Celui des soignantes d'abord. Puis le propre regard de Mme B dans le miroir mural de l'unité. Miroir duquel elle n'est résolument plus absente, mais bel et bien présente : elle perçoit et contemple son propre reflet, et se trouve belle avec son nouveau maquillage. Mme B redevient ainsi présente à sa propre image, et surtout elle semble de nouveau se sentir digne d'intérêt pour elle-même et pour l'autre. Une réhumanisation qui, en modifiant le rapport qu'elle entretient d'elle à elle, vient immanquablement modifier son rapport à "ses bébés " (réels, imaginaires... mais aussi peut-être le bébé en elle) et ainsi transformer les relations mère-enfants. C'est du fait d'un rapportcharnière avec l'équipe soignante, alors représentant symbolique de la société, qu'une seconde hypothèse peut être émise en guise de conclusion : c'est en prenant soin de la relation mère/équipe-soignante et donc symboliquement mère/société, que l'on peut véritablement prendre soin de la relation mèreenfant.

\section{RÉFÉRENCES}

Cabut S. et Santi P. (2017). Les enfants sans domicile en première ligne. Le Monde "Science et médecine ", Numéro du mercredi 15 mars 2017.

Ciccone A. (2012). La pratique de l'observation. Contraste, $\mathrm{n}^{\circ} 36,55-77$.

Ciccone A. et Lhopital M. (2001). Naissance à la vie psychique. Paris, Dunod (2e ed).

Deronzier D. (2015). L'avidité et ses destins, à partir de l'œuvre de D.W. Winnicott. Fournal de la psychanalyse de l'enfant, 5(2), 143-158.

Devereux G. (1967). De l'angoisse à la méthode dans les sciences du comportement. Paris : Aubier, 1994.

Ferrant A. (2004). Le regard, la honte et le groupe. Revue de psychothérapie psychanalytique de groupe, $\mathrm{n}^{\circ} 43,145-158$.

Furtos J. (2008a). L'apparition du sujet sur la scène sociale et sa fragilité : la précarité de la confiance. Dans J. Furtos (dir.), Les Cliniques de la précarité : contexte social, psychopathologie et dispositifs (p. 11-22). Issy-Les-Moulineaux : Elsevier Masson.

Furtos J. (2008b). Le syndrome d'auto-exclusion. Dans J. Furtos (dir.), Les Cliniques de la précarité : contexte social, psychopathologie et dispositifs (p. 118-133). Issy-LesMoulineaux : Elsevier Masson.

Furtos J. (2008c). Conclusions générales : angoisse de non-assignation et santé mentale. Dans J. Furtos (dir.), Les Cliniques de la précarité : contexte social, psychopathologie et dispositifs (p. 278-284). Issy-Les-Moulineaux : Elsevier Masson.

Green A. (1983). La mère morte. Dans A. Green, Narcissisme de vie, narcissisme de mort (p. 222-254). Paris : Les Éditions de Minuit.

Green A. (1999). Passivité-passivation : jouissance et détresse. Revue française de psychanalyse, 63(5), 1587-1600.

Guyavarch E. et Garcin E. (2014). Public hébergés par le 115 de Paris : une forte progression des familles. Informations sociales, $\mathrm{n}^{\circ} 182,142-149$.

Kaës R. (2014). Les Théories psychanalytiques du groupe. Paris : Puf, "Que Sais-je ?".

Mansouri M. et Bentata H. (2012). Sans famille, sans papiers : des bébés et leurs mères enfermés dehors? Dans C. Davoudian (dir.), Mères et bébés sans-papiers (p. 115131). Paris : Érès.

Meryglod N. (2008). Une clinique de l'incurie. Dans J. Furtos (dir.), Les Cliniques de la précarité : contexte social, psychopathologie et dispositifs (p. 182-194). Issy-LesMoulineaux : Elsevier Masson.

Moro M.R. (1998). Psychothérapie transculturelle des enfants de migrants. Paris : Dunod.

Moro M.R. (2002). Enfants d'ici venus d'ailleurs : naitre et grandir en France. Paris : La Découverte. 
Paillé P. (1994). L'analyse par théorisation ancrée. Cahiers de recherche sociologique, $n^{\circ} 23,147-181$.

Rochette J. (2008). Précarité et périnatalité précoce : 40 jours pour transformer le désordre aléatoire en chaos organisé. Dans J. Furtos (dir.), Les Cliniques de la précarité : contexte social, psychopathologie et dispositifs, (p. 98-113). Issy-Les-Moulineaux : Elsevier Masson.

Rouchon J.-F., Reyre A., Taïeb O., et Moro M.R. (2009). L'utilisation de la notion de contre-transfert culturel en clinique. L'Autre, 10(1), 80-89.

Roussillon R. (2005a). Les situations extrêmes et la clinique de la survivance psychique. Dans J. Furtos et C. Laval (dir.), La Santé mentale en actes (p. 221-238). Toulouse : érès.

Roussillon R. (2005b). Le rythme, le transitionnel, le transit et le cadre : à propos d'une forme de traumatisme " qualitatif ". Dans R. Roussillon, Paradoxes et situations limites de la psychanalyse (p. 198-217). Paris : Puf, "Quadrige».

Roussillon R. (2008b). La loi du plus faible : les stratégies de survie. Dans J. Furtos (dir.), Les Cliniques de la précarité : contexte social, psychopathologie et dispositifs (p. 134138). Issy-Les-Moulineaux : Elsevier Masson.

Schmit G. et Soulé M. (2004). L'énurésie infantile. Dans S. Lebovici et al., Nouveau traité de psychiatrie de l'enfant et de l'adolescent (pp. 1751-1770). Paris : Puf, "Quadrige".

Winnicott D.W. (1957). Le monde à petite dose. Dans D.W. Winnicott, L'Enfant et sa famille (p. 89-96), trad. fr. A. Stronk-Robert. Paris : Payot, 2002.

Zaltzman N. (1999). La pulsion anarchiste. Dans N. Zaltzman, De la guérison psychanalytique (p. 107-156). Paris : Puf (2 éd.).

Automne 2018

Rebecca Faura-Loussert 15, rue Neuve-des-Boulets 75011 Paris rebecca.fauraloussert@gmail.com 